\title{
HEPATIC STELLATE CELLS ON POLY(DL-LACTIC ACID) SURFACES CONTROL THE FORMATION OF 3D HEPATOCYTE CO-CULTURE AGGREGATES IN VITRO
}

\author{
Thomas RJ, Bennett $\mathrm{A}^{1}$, Thomson $\mathrm{B}^{2}$, and Shakesheff KM* \\ Tissue Engineering Group, School of Pharmacy, The University of Nottingham, NG7 2RD, UK. ${ }^{1}$ School of \\ Biomedical Sciences \& ${ }^{2}$ School of Molecular Medicine
}

\begin{abstract}
Evidence for the functional superiority of cells cultured as $3 \mathrm{D}$ aggregates or on 3D scaffolds over conventional 2D monolayer cultures has created interest in material and cell based methods that influence the formation and structure of multicellular aggregates in vitro. We have created a coculture of primary rat hepatocytes and hepatic stellate cells on a poly(DL-lactic acid) surface, a poor substrate for rat hepatocyte adhesion, to study the dynamics of multicellular spheroid formation and the resultant cell arrangement. The poly(DL-lactic acid) surface allows dynamic and rapid interaction of hepatocytes and stellate cells to form coculture spheroids in a complex multistage process (shown by time lapse microscopy). This spheroid morphology supports enhanced cell viability relative to a mono-culture mono-layer system (measured by lactate dehydrogenase leakage). The distribution of the aggregating cell type in the final structure is related to the mechanics of formation i.e. mainly central and peripheral. This study provides a unique and generically applicable insight into the dynamics of multicellular spheroid formation where aggregation is induced by one cell type and imposed on another. This has implications for 3D cell culture models and a wide number of currently used stromal co-culture systems.
\end{abstract}

Key Words: Poly(DL-lactic acid), stellate cell, Hepatocyte, Tissue culture, 3D

\author{
Abbreviations used: \\ SMA - a smooth muscle actin \\ GFAP - Glial fibrillary acidic protein \\ LDH - Lactate dehydrogenase \\ $\mathrm{P}_{\mathrm{DL}}$ LA - Poly(DL-lactic acid)
}

\section{Introduction}

Improved cell culture techniques are required to provide a source of cells for tissue based therapies as well as reliable in vitro experimental models for studies of infection, pathogenesis, toxicology and other areas. In many fields of in vitro cell culture multicellular 3D structures offer improved in vivo like function, morphology and responses in comparison to conventional 2D mono-layer cultures (Schmeichel and Bissell, 2003). These models are inherently complex and often involve multiple cell types and heterogenous morphology. The connection between cell arrangement and function has generated interest in different methods of cell organisation in vitro.

A multicellular 3D structure is thought to be advantageous in cell culture due to the reformation of in vivo-like 3D architecture and associated cell-cell and cellmatrix connectivity. Hepatocyte 3D aggregate structures have more cadherin expression and less integrin expression than monolayers suggesting more cell to cell communication (Hou et al., 2001). Unsurprisingly, increased cell to cell connectivity in vitro has been linked to improved cell function (Brieva and Moghe, 2001). Enhanced liver specific function, hepatocyte membrane polarity and aspects of liver ultrastucture, such as cellular junctions and bile canaliculi, are commonly reported in 3D culture systems including aggregate cultures in bioreactors (Zeilinger et al., 2004; Powers et al., 2002), collagen sandwich cultures (Engl et al., 2004), spheroid cultures (Koide et al., 1990; Abu-Absi et al., 2002; Kudryavtseva and Engelhardt, 2003; Dvir-Ginzberg et al., 2004), and cultures on polymer scaffolds (Hanada et al., 2003).

There is evidence that the method and mechanism of hepatocyte aggregation has importance in the functional performance of the resultant cell structure. Growth factor supplementation and altered matrix compliance have been shown to change the kinetics of aggregation (Semler et al., 2000), and variation of culture surface properties to influence the biological mechanism of spheroid formation (Hasebe et al., 2005), both with functional consequences. The speed of aggregation and, in co-cultures, the arrangement of cells with respect to heterotypic interfaces (Bhatia et al., 1998) are also thought to be important in determining a systems final functional characteristics. In this study the dynamics of formation and resultant structure of 3D hepatocyte - hepatic stellate cell co-culture spheroids on a low adherence $\mathrm{P}_{\mathrm{DL}} \mathrm{LA}$ surface are explored. This system possesses significant functional benefits compared with a 2D hepatocyte monoculture (Bhandari et al., 2001, Thomas et al., 2006) and is therefore an important model of functionally beneficial aggregation. 


\section{Materials and Methods}

\section{Cell Isolation and culture}

\section{Rat Hepatocyte Isolation}

Hepatocytes were isolated from male Wistar rats weighing approximately $180 \mathrm{~g}$ using the two-step collagenase perfusion described by Seglen, with minor modifications (Seglen, 1976). In brief, rats were killed by cervical dislocation and the livers were perfused with $0.5 \mathrm{mM}$ EGTA in Hanks-Hepes buffer for approximately 15 minutes, followed by $5 \mathrm{mM}$ calcium and $100 \mathrm{U} / \mathrm{ml}$ Type IV collagenase enzyme (Sigma) in Hank's-Hepes buffer until digested. After isolation and washing, hepatocytes were purified from non-viable and non-parenchymal cells by centrifugation at $50 \mathrm{~g}$ for 5 minutes in a defined density media (45\% Percoll (Sigma, density 1.125-1.135 g/ml), 5\% 10x Hank's buffered salt solution, 45\% William's E Media (Gibco), 5\% foetal calf serum (Sigma)). Viability was determined by Trypan blue exclusion, and was typically above $90 \%$.

\section{Rat Stellate cell isolation}

Stellate cells were isolated as described previously (Riccalton-Banks et al., 2003). In brief, supernatant from cell washes and the percoll purification spin were combined, and made up to $200 \mathrm{ml}$ with stellate culture media (DMEM (Gibco) supplemented with $5 \mathrm{mM} \mathrm{L-}$ glutamine, $100 \mathrm{U}$ Penicillin, $100 \mathrm{mg}$ streptomycin, and $10 \%$ FCS Gold (PAA laboratories)). This mixture was centrifuged three times at $50 \mathrm{~g}$, each time retaining the supernatant and discarding the pellet. The final supernatant was centrifuged at $260 \mathrm{~g}$, the resultant pellet suspended in stellate culture medium, and plated into two T75 flasks. Media was changed after 12 to 24 hours and then every three days. Cells were cultured for several weeks until confluent before use.

\section{$\mathbf{P}_{\mathrm{DL}}$ LA coating of culture plates \\ $\mathrm{P}_{\mathrm{DL}}$ LA (Alkermes Inc., USA) was dissolved in 2,2,2- Trifluoroethanol at a concentration of $1.5 \mathrm{mg} / \mathrm{ml} .500 \mathrm{ml}$ of $\mathrm{P}_{\mathrm{DL}}$ LA solution was added to each $962 \mathrm{~mm}^{2}$ Nunclon dish. The dishes were placed in an oven at $60^{\circ} \mathrm{C}$ for approximately 1 hour to evaporate the solvent and then exposed under a sterilising UV lamp for 30 minutes before being stored at $-20^{\circ} \mathrm{C}$.}

\section{Cell culture and experimental design}

Co-cultures comprising of 300000 cultured stellate cells and 600000 freshly isolated hepatocytes, HepG2 cells or A549 cells were combined in a $\mathrm{P}_{\mathrm{DL}}$ LA coated $962 \mathrm{~mm}^{2}$ plate, and maintained in $1.5 \mathrm{ml}$ hepatocyte culture medium (William's E media supplemented with $5 \mathrm{mM} \mathrm{L}$-glutamine, $50 \mathrm{mg} / \mathrm{ml}$ gentamicin, $5 \mathrm{mM}$ nicotinamide, and $10 \mathrm{mU} / \mathrm{ml}$ insulin) at $37^{\circ} \mathrm{C}$ in a $5 \% \mathrm{CO}$, atmosphere. Mono-cultures were prepared with either 600000 hepatocytes or 300000 stellate cells and incubated in the same way. Hepatocyte fragments were prepared by briefly sonicating a primary hepatocyte suspension and then centrifuging out large particles and whole cells at $50 \mathrm{~g}$ for 5 minutes. The supernatant was added to a mono-culture of 300000 stellate cells. A high density area of stellate cells, confirmed by microscopy, was created by allowing a $30 \mathrm{ml}$ drop containing 300000 stellate cells to sit in a plate for 10 minutes before subsequent normal seeding. All culture types were studied by time-lapse photography. Cell death was analysed in duplicate hepatocyte mono-culture and hepatocyte - stellate cell co-cultures. Cell organisation was studied in multiple 5 day old hepatocyte - stellate cell coculture sections.

\section{Time Lapse Microscopy}

Cells were kept in a $37^{\circ} \mathrm{C} \mathrm{CO}$, supplemented incubation chamber under a Leica DR IRBE microscope with an attached Leica DC 200 digital camera. Images were acquired every 6 minutes and converted into video using Adobe Premier ${ }^{\mathrm{TM}}$ software.

\section{LDH assay}

The LDH assay was conducted using a commercially available kit (Roche applied science - Cytotoxicity detection kit (LDH)). Supernatant was removed from the cells and centrifuged to remove any cells/spheroids and then refrigerated. Any pelleted cells/spheroids were lysed using $0.5 \mathrm{ml}$ Triton-X 100 (1\%) in assay medium that was then transferred to lyse the remaining cells in the culture well. The assay was conducted in a 96 well plate using $100 \mathrm{ml}$ of assay supernatant or cell lysate and $100 \mathrm{ml}$ of freshly prepared Diaphorase, $\mathrm{NAD}^{+}$, Iodotetrazolium chloride and sodium lactate mixture (from kit). Incubation was 30 minutes. Formazan formation (directly related to LDH content) was measured by detecting absorbance in a plate reader at $490 \mathrm{~nm}$. Percentage cell death was calculated by dividing the LDH activity value from the supernatant by the LDH activity value of the supernatant and remaining cell lysate combined and multiplying by 100 .

\section{Spheroid Sectioning and Immunohistology}

5 day old spheroids or liver samples were prepared for histology by fixing tissue in $10 \%$ formalin for approximately 1 hour, suspending the fixed tissue in an agarose gel pellet, and then embedding the pellet in paraffin. $4 \mathrm{~mm}$ sections were then cut, dehydrated, incubated at $4{ }^{\circ} \mathrm{C}$ with a 1:250 dilution of Sigma monoclonal anti-aSMA antibody (clone 1A4), followed by a FITC labelled anti-mouse $\mathrm{IgG}$, rehydrated, and mounted in DAPI/DPX.

\section{Results}

Hepatocytes co-cultured with stellate cells on a $\mathrm{P}_{\mathrm{DL}} \mathrm{LA}$ surface formed spheroids of $100-150 \mu \mathrm{m}$ in diameter over approximately two days. If a gradient of stellate cell density was present spheroids formed only in areas of high stellate cell concentration with hepatocyte monolayer formation in areas of lower stellate cell density (Figure 1).

The lowest magnification hepatocyte-hepatic stellate cell co-culture time lapse movie gives a temporal overview of the course of aggregation and spheroid formation (Figure 2, Time lapse 1). The process occurred in several 

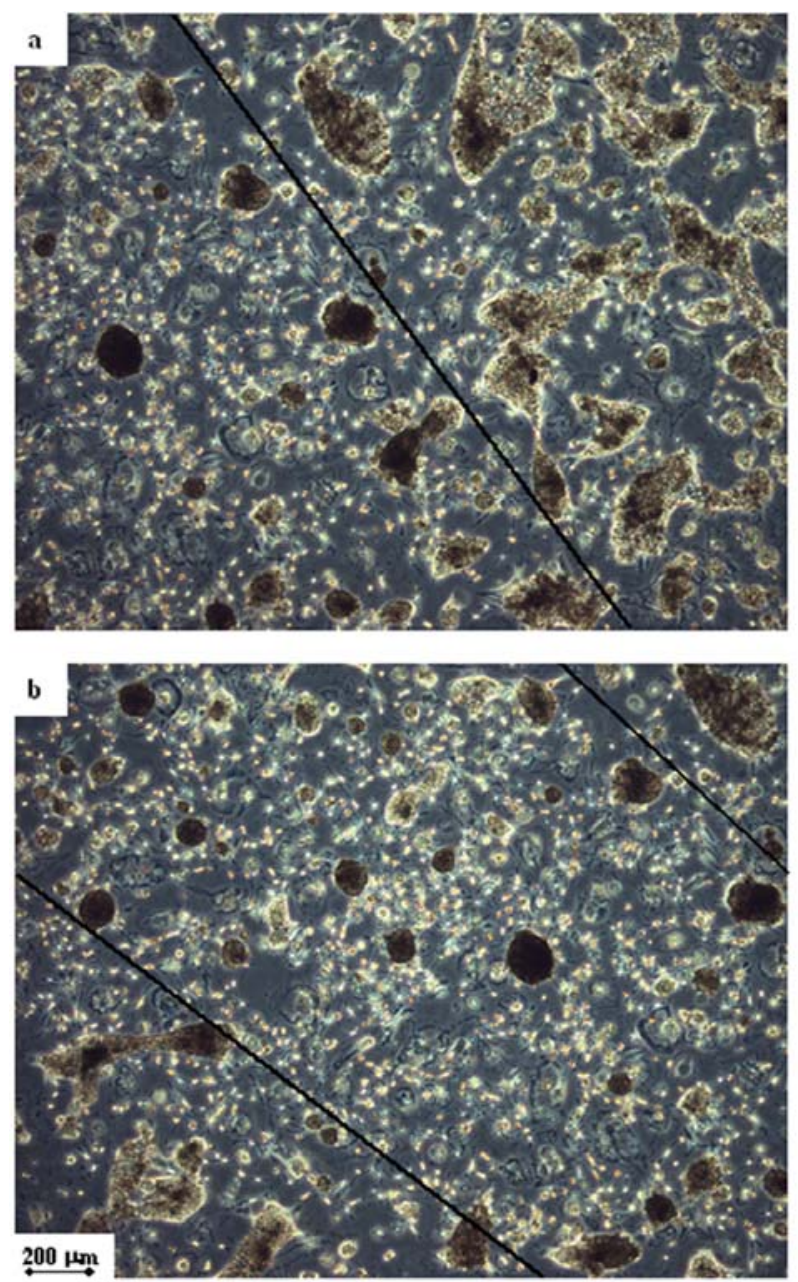

Figure 1: These images show that when a gradient of stellate cell concentration was presented, co-culture spheroid formation occurred only in areas of high stellate cell density. The area of high stellate cell density, and consequent spheroid formation, is marked to the left of the line in image (a) and in between the lines in image (b)). Outside these areas, where stellate cell density was lower, hepatocyte attachment to the culture surface occurred.

distinct phases, each of which is annotated on the timelapse movies (to be found through links on the paper web page-http://www.ecmjournal.org/journal/papers/vol011/ vol011a0x.php). The first 12 hour period of culture was characterised by low cell motility and some hepatocyte spreading. This was followed by 12 hours of relatively high cell motility in which rapid stellate cell contraction led to aggregate formation. A further 12 hours of consolidation occurred in which the outlines of the spheroids became smooth and distinct, and the remaining cells were amalgamated. After this phase the spheroids maintained a stable morphology.

The highest magnification hepatocyte-hepatic stellate cell co-culture time lapse movie provides a detailed view of the heterotypic cell interactions (Figure 3, Time lapse 2). Stellate cells rapidly develop the normal culture activated multiple process morphology (Sato et al., 2003) in the first few hours of culture. Throughout the first 8 hours of culture, despite hepatocyte contact, contraction to aggregates does not occur. After the first 8 hours of culture the stellate processes rapidly retract creating coculture aggregates. After 20-24 hours the aggregates undergo some consolidation. The tension developed in the stellate cell contractile response can be seen at the beginning of this period (in the top left of the movie) as a spheroid is literally catapulted off screen by a stellate process. This movie clearly shows the stellate cells to be highly motile and the hepatocytes to be immobile except under the active contractile control of the stellate cells.

The stellate cells did not display the same level of motility or retract their processes in the same manner when mono-cultured on a $\mathrm{P}_{\mathrm{DL}}$ LA surface (Figure 4, Time lapse 3 ). However they did retract their processes rapidly in response to hepatocyte conditioned media (Figure 5, Time lapse 4). Also, stellate cells exhibited the same contractile response to hepatocyte fragments as whole cells (Figure 6 , Time lapse 5). This aggregation occurred as soon as the cell fragments were added. Stellate cells partially aggregated epithelial liver cell line Hep G2 cells but slower and with formation of less well defined aggregates than primary hepatocytes (Figure 7, Time lapse 6). Stellate cells failed to aggregate epithelial pulmonary cell line A549 cells (not shown).

Hepatocytes were static and did not adhere to the $\mathrm{P}_{\mathrm{DL}} \mathrm{LA}$ culture surface or form aggregates when mono-cultured on $\mathrm{P}_{\mathrm{DL}} \mathrm{LA}$ (Figure 8, Time lapse 7). All of the culture types were conducted many times with consistent results.

A study of the viability of the co-culture spheroids relative to cells in mono-culture demonstrated a stabilisation of cell viability after spheroid formation (Figure 9). Between 24 and 48 hours of culture cell death assessed by LDH leakage was approximately $10 \%$ in both mono-culture and co-culture. However, between day 2 and day 5 of culture, $30 \%$ of remaining cells in co-culture died relative to $58 \%$ in mono-culture. A similar result of $24 \%$ to $49 \%$ respectively was observed between day 5 and day 7.

The immunological characteristics of the stellate cell population were studied in mono-culture after a protracted period of approximately 3 weeks in vitro and immediately prior to incorporation into co-culture. The presence of GFAP and desmin confirmed the identity of the stellate cells and SMA demonstrated an activated morphology (not shown). After 5 days in co-culture the stellate cells were identified in spheroid sections by immunolocalisation of SMA. They were located mainly around the periphery and centre of the spheroids (figure 10).

\section{Discussion}

3D cell aggregate formation is an effective method of improving tissue function in vitro. However, the manner and mechanism of aggregate formation as well as the final aggregate structure are influential in maximising functional benefits. Here we discuss the properties of a hepatocytehepatic stellate cell co-culture on a $\mathrm{P}_{\mathrm{DL}} \mathrm{LA}$ surface as a method of controlling hepatocyte aggregation through heterotypic cell interaction. 

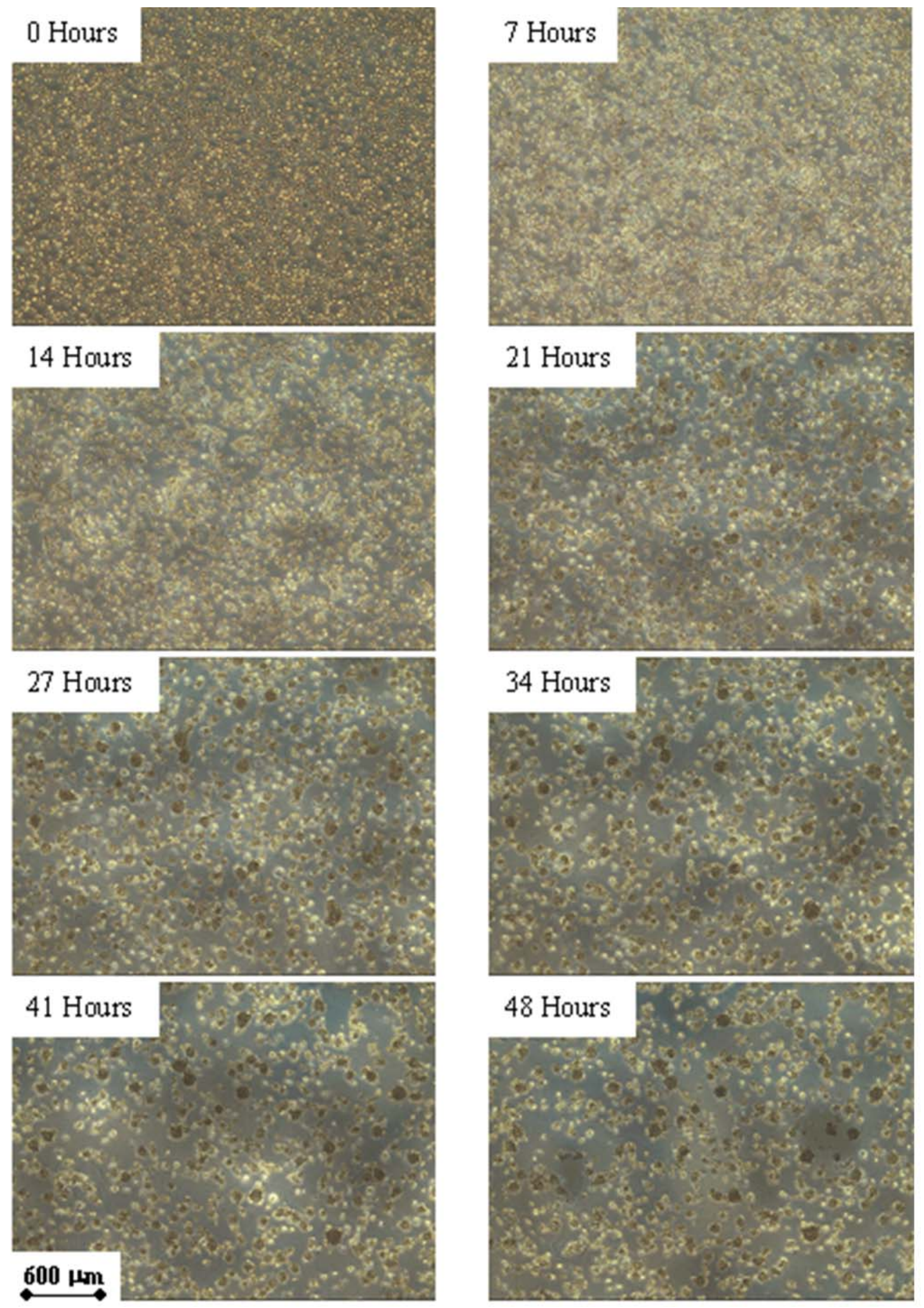

Figure 2: A sequence of images from time lapse movie 1 shows formation of co-culture spheroids. The stellate cell motility, initially low, increased with time in culture with hepatocytes. Spheroid formation occurred rapidly in an approximately 6 hour window in the region of 15 hours into co-culture. After this time spheroids were stable (for actual movie, see the main paper Web page). 

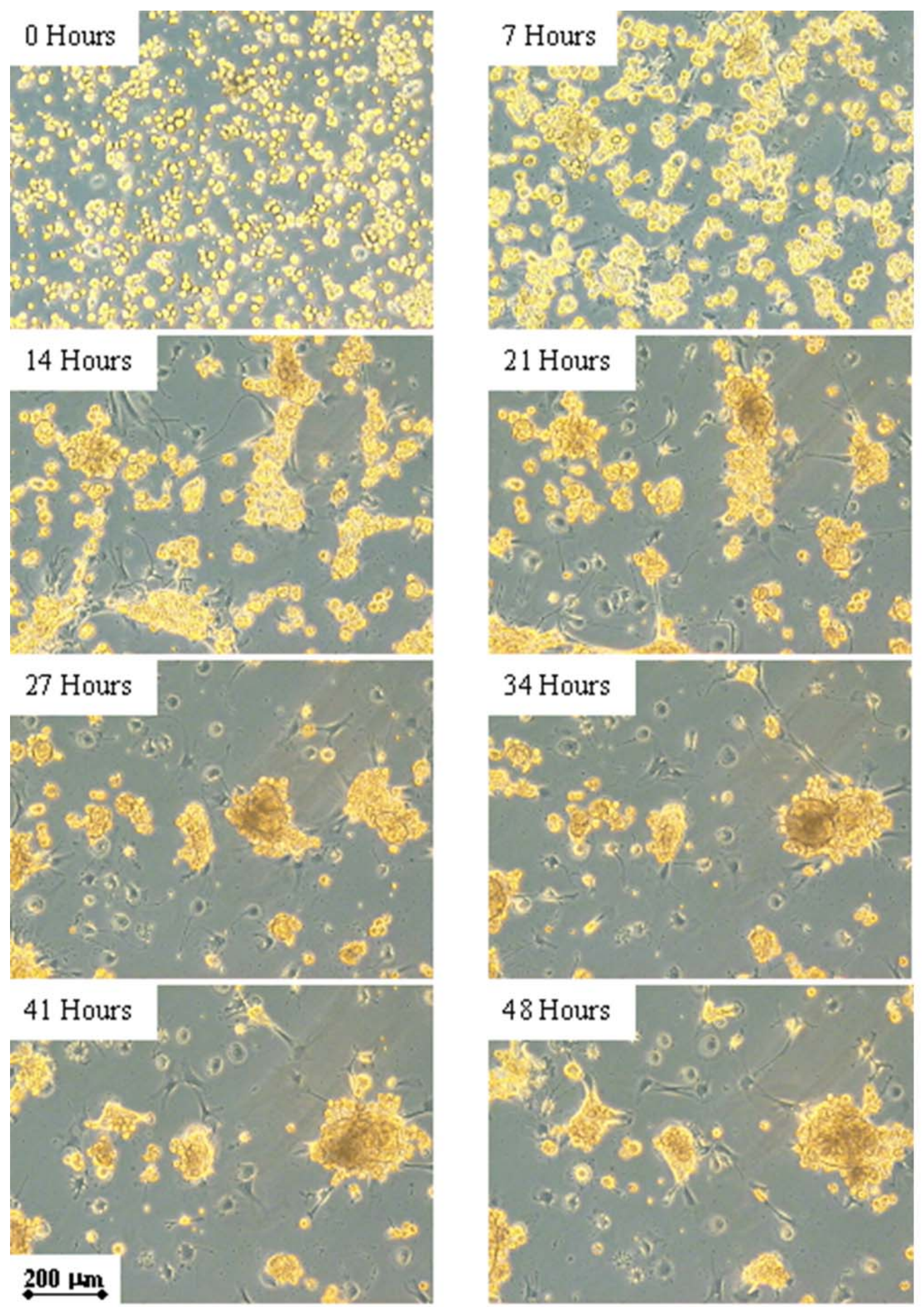

Figure 3: A sequence of images from time lapse movie 2 shows formation of co-culture spheroids. The spheroid processes contracted when contacted by hepatocytes. The speed and contractility increased with time in culture resulting in rapid spheroid formation in the same time window as described in figure 2 . The top left of the movie at 21 hours shows a newly formed spheroid under tension from a contracting stellate process that is pulled off screen shortly after (for actual movie, see the main paper Web page). 

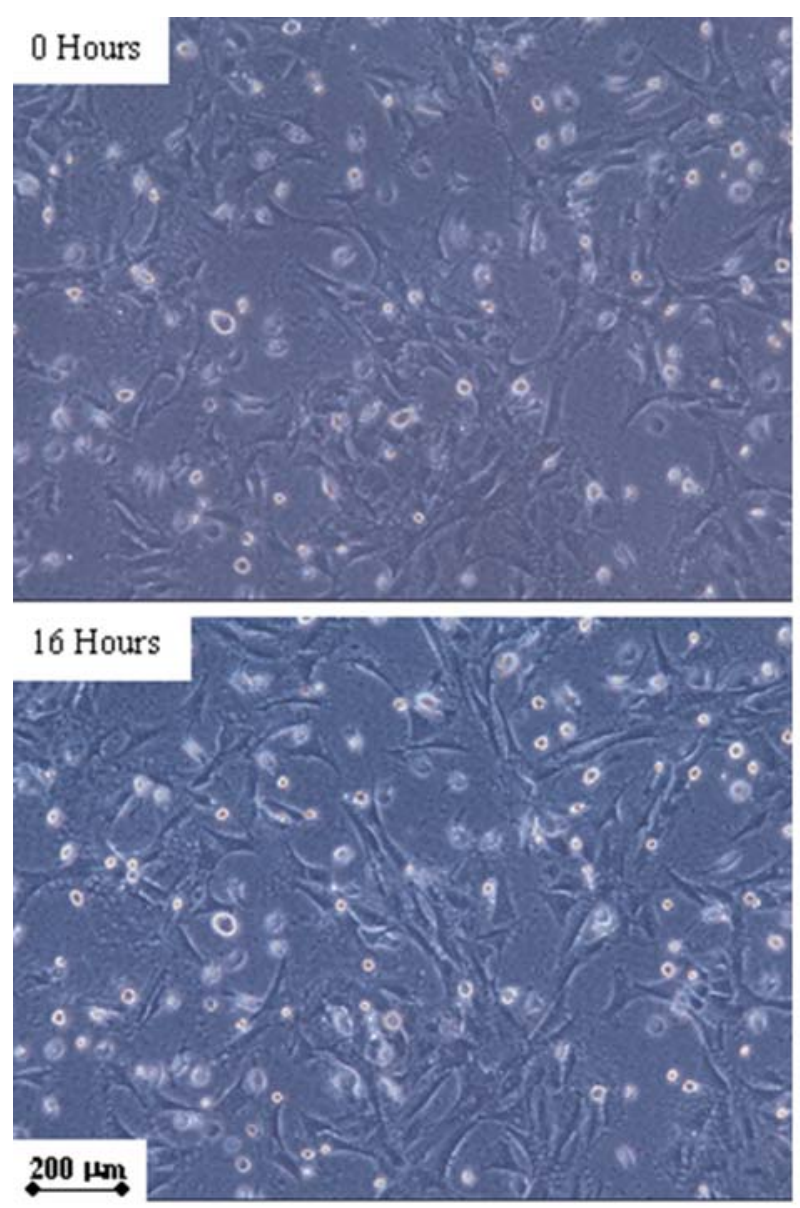

Figure 4: A sequence of two images from time lapse movie 3 shows the activated stellate cell processes did not contract, and stellate cell motility was relatively low, in mono-culture after a similar period in culture to that at which maximum motility was observed in co-culture (for actual movie, see the main paper Web page).
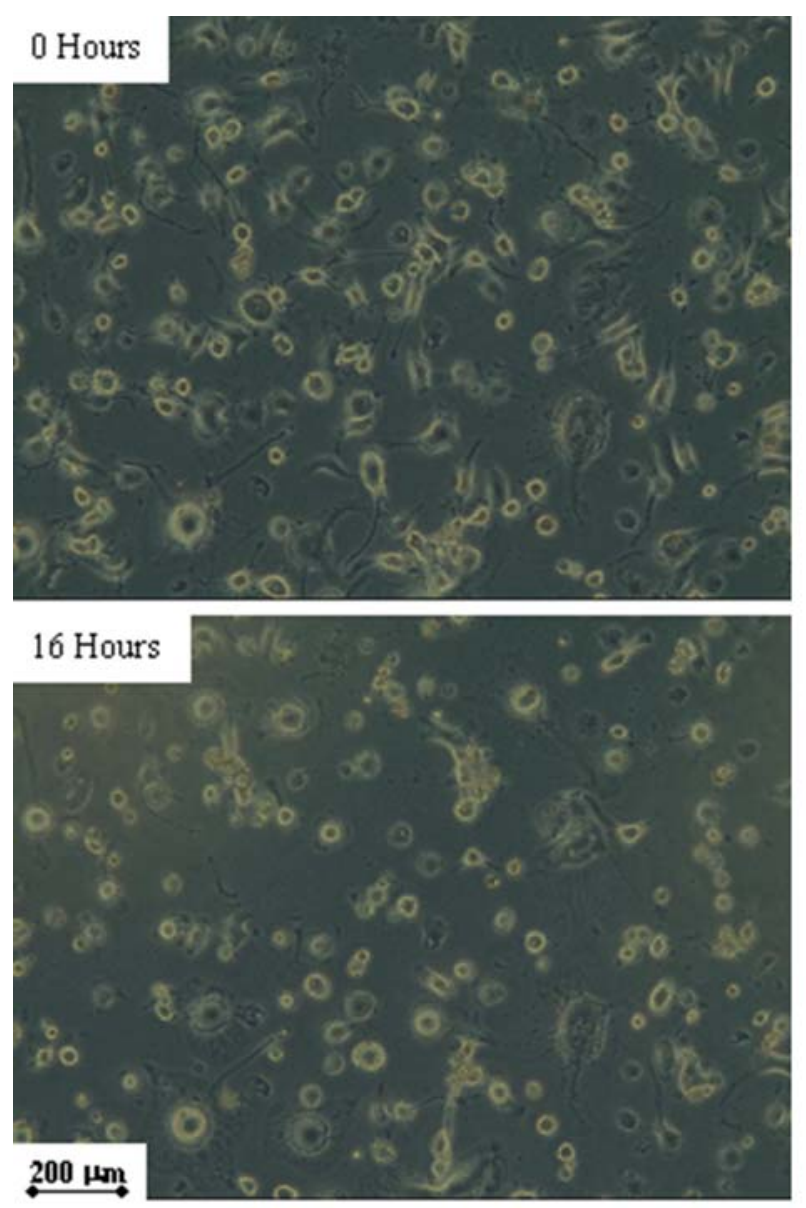

Figure 5: A sequence of two images from time lapse movie 4 shows the activated stellate cells in monoculture rapidly retracted processes in response to hepatocyte conditioned media (for actual movie, see the main paper Web page).
In hepatocyte stellate cell co-culture, aggregation is not a result of passive cell collision and adhesion. It is an active process dependent on stellate cell motility. The stellate cell process contraction and morphology is influenced by hepatocyte co-culture and this process is informative about the factors influencing cell aggregation. The delay before the start of stellate cell process contraction, despite cell contact, and the increase in stellate cell process contractility over time, has a number of potential explanations including adaptation of gene expression leading to build up of cell surface protein or soluble mediator expression. Formation of co-culture spheroids only in areas of high stellate cell density, combined with the failure of stellate cells to facilitate alternative epithelial cell line spheroid formation as effectively as primary hepatocyte spheroid formation, suggests factors from both stellate cells and differentiated hepatocytes are required for stellate facilitated spheroid formation. The contraction of stellate cells in response to hepatocyte fragments and hepatocyte conditioned media supports the involvement of a hepatocyte soluble mediator(s) and eliminates the involvement of complex cross talk between the two cell types (i.e. a signal from one cell type triggering a signal from the second cell type that then acts on the first) in eliciting this response.

The structure of the co-culture produced spheroids is related to the mechanism of formation. Stellate cells on the periphery of the spheroids are hypothesised to be those that were beneath the hepatocytes during the initial plating phase and therefore end up on the exterior of the spheroid; these are often thicker on a flatter side of a spheroid, presumably the side that was on the culture surface. When a stellate cell is located in the centre of a spheroid this is thought to be the result of the stellate cell attaching on the top of hepatocytes, or pulling two smaller aggregates together. The benefit of this type of aggregation in which one cell type actively pulls a second passive cell type around it is seen in the high degree of heterotypic contact. The improved maintenance of cell viability in co-culture relative to mono-layer only occurs after the cells have adopted the multicellular spheroid morphology. This suggests that fast aggregation could be important in preventing early cell death. Also, hepatocytes in contact with a heterotypic cell type have greater function than their 

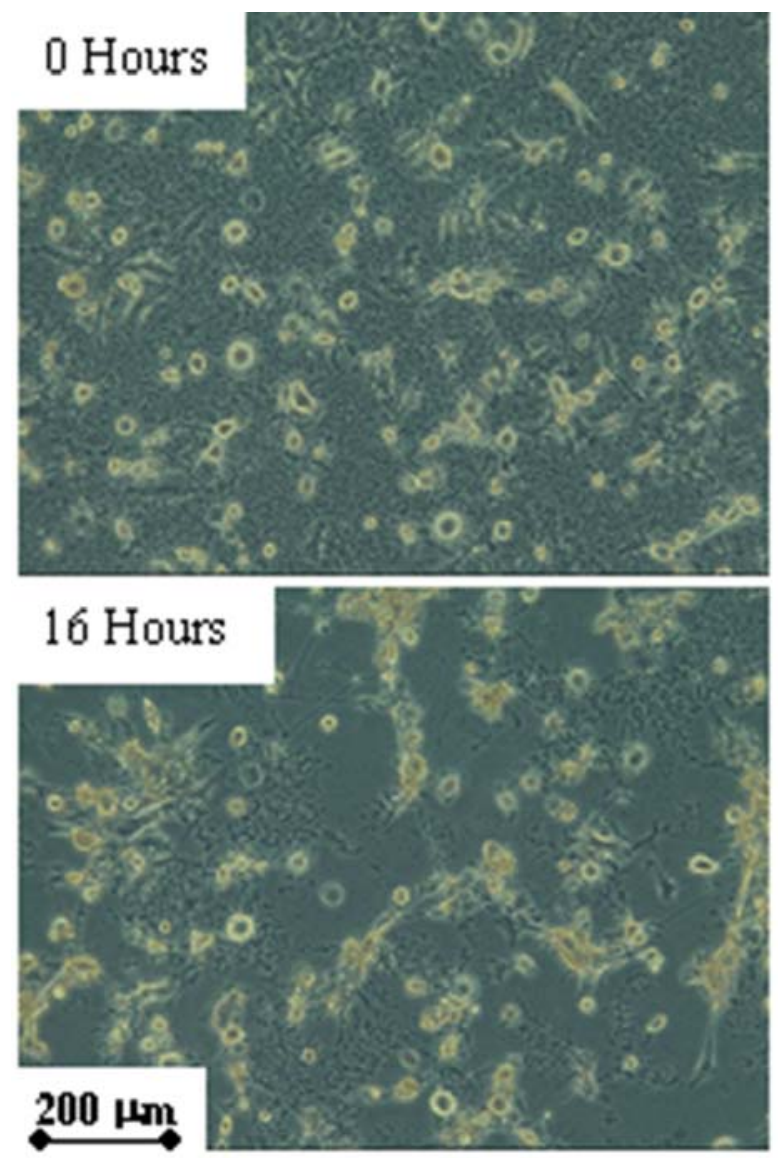

Figure 6: A sequence of images from time lapse movie 5 shows activated stellate cell processes contracted in response to hepatocyte fragments (added immediately prior to imaging) (for actual movie, see the main paper Web page).

isolated counterparts (Bhatia et al., 1998). In combination this suggests that methods such as this that rapidly establish heterotypic cell contact and maximise heterotypic cell interfaces through 3D morphology are an important tool in producing viable and functional tissue aggregates.

Co-culture on a $\mathrm{P}_{\mathrm{DL}} \mathrm{LA}$ surface demonstrates an optimised environment for cell interaction, aggregation and associated benefits. The process is complex and probably involves both stellate cell and hepatocyte derived mediators, although not cellular cross talk. This study provides a generic model of aggregation and cellular arrangement for multicellular structures where formation is mediated by the contractility of one cell type. Such methods have a potentially important role in functional and structural support of tissue engineered cell culture aggregates, and provide a valuable method of aggregating non-motile cells.

\section{Acknowledgments}

BBSRC for funding of $\mathrm{PhD}$ Studentship. $\mathrm{AB}$ thanks FRAME.
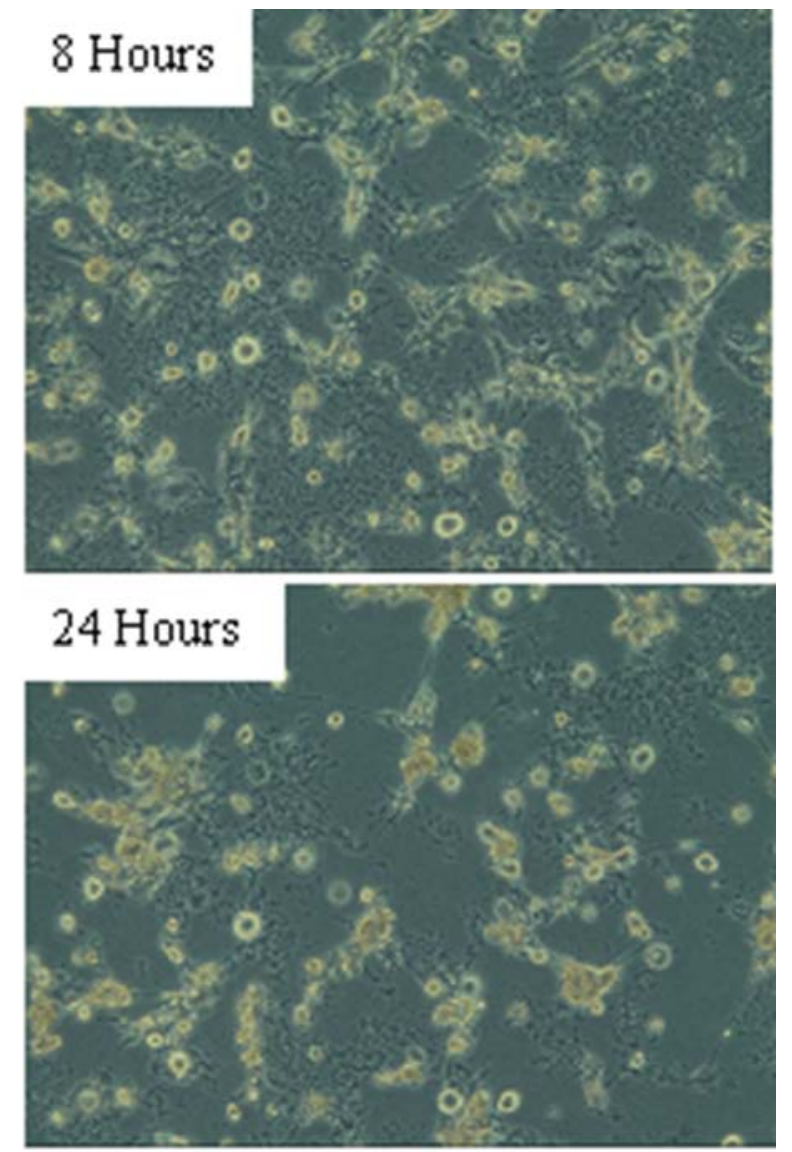

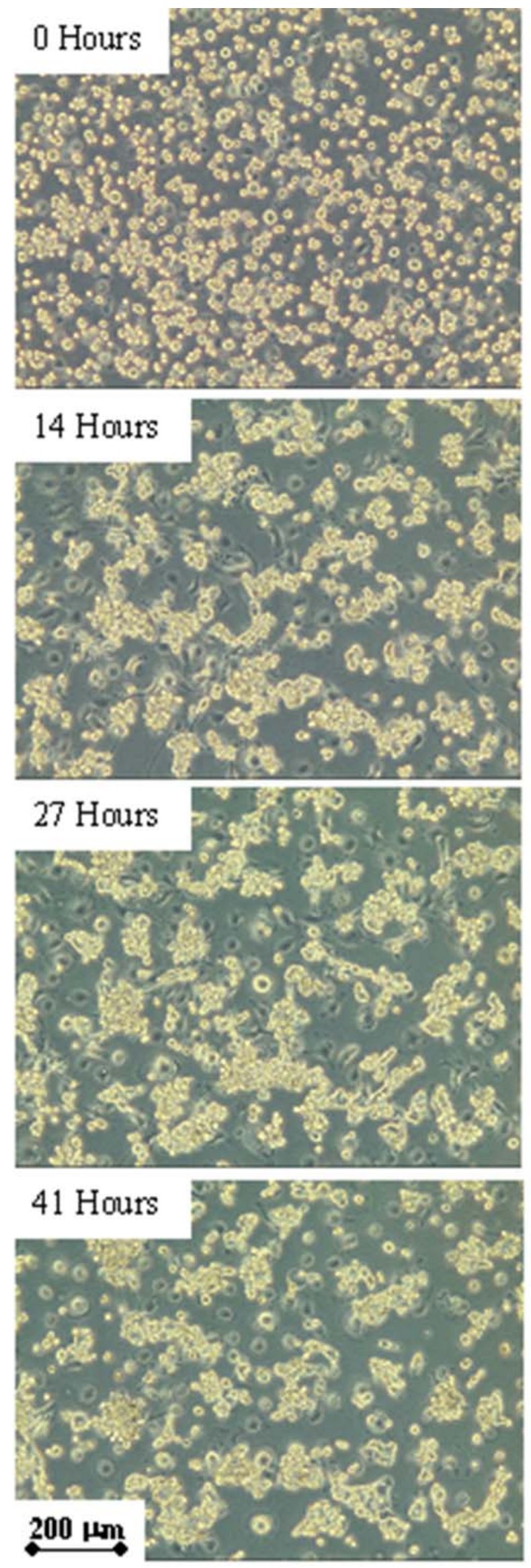
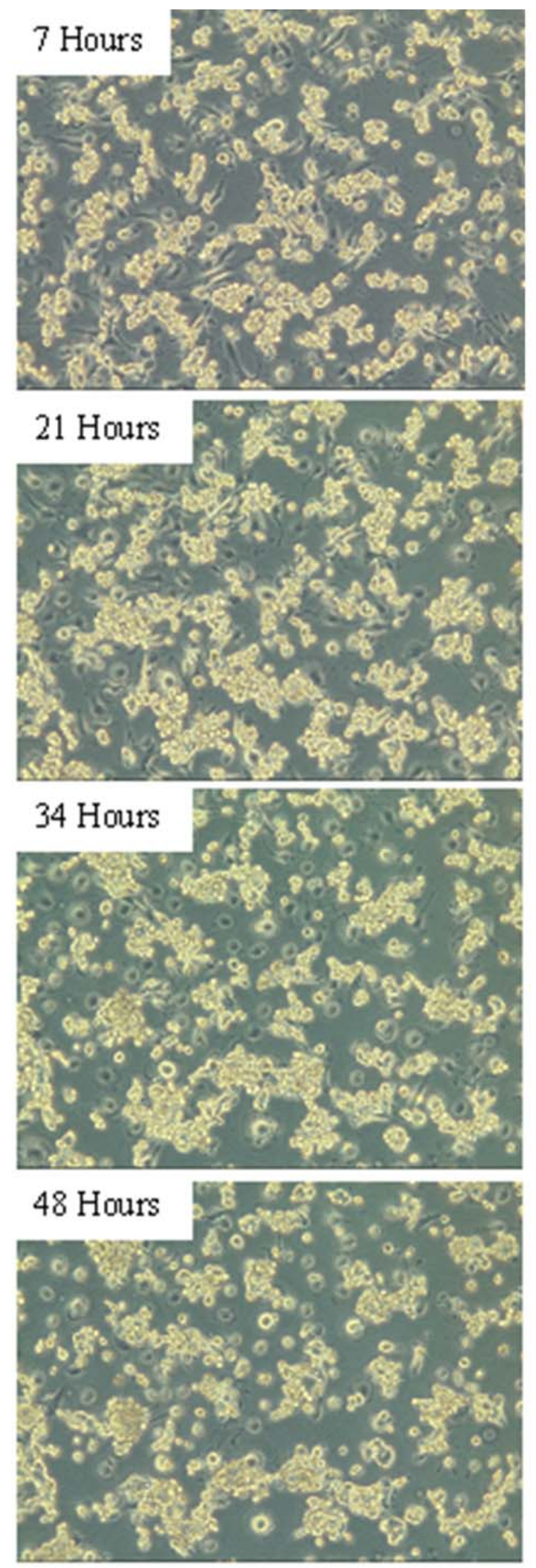

Figure 7: A sequence of images from time lapse movie 6 showing activated stellate cells fail to aggregate Hep G2 cells in the same manner as freshly isolated primary hepatocytes (for actual movie, see the main paper Web page). 

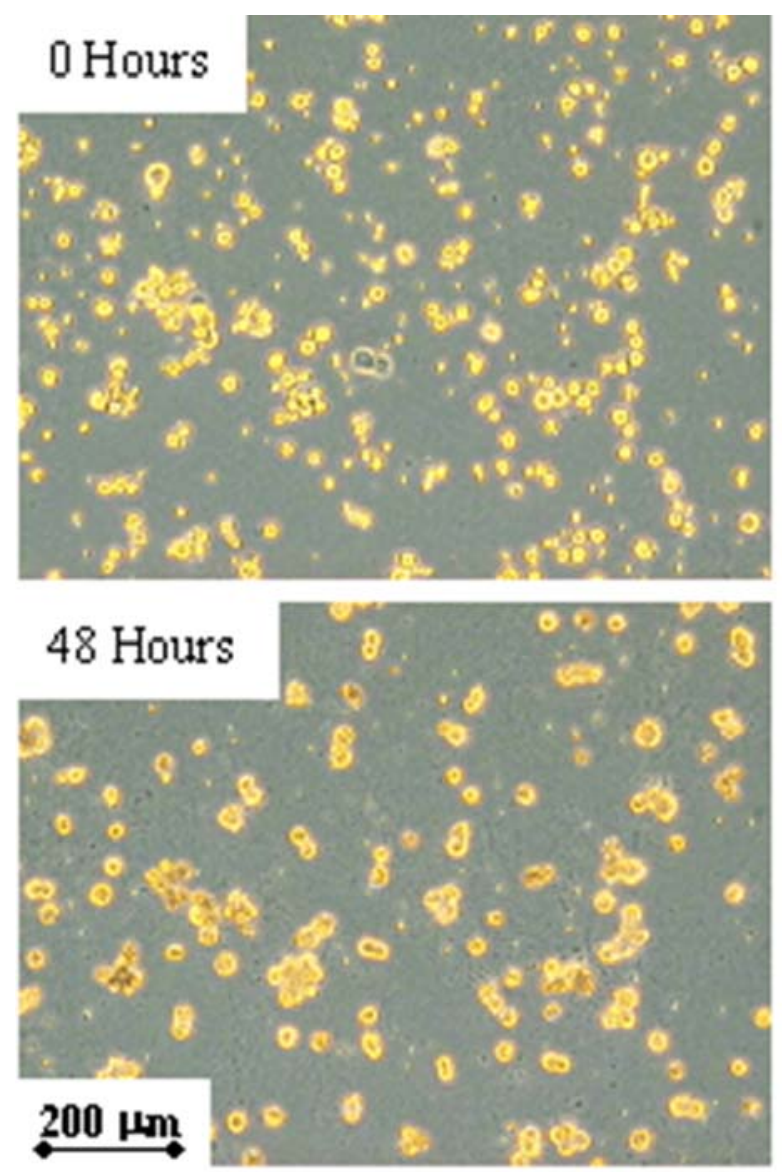

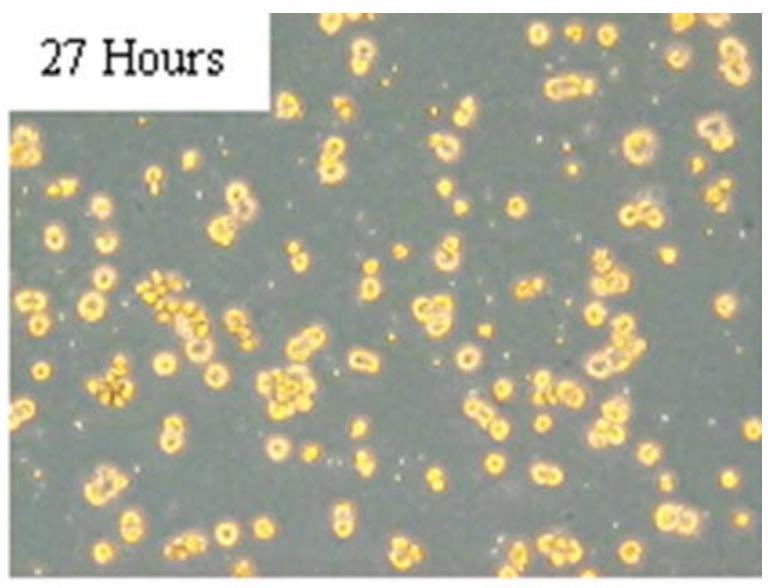

Figure 8: A sequence of images from time lapse movie 7 showing hepatocytes do not aggregate or attach to the surface when mono-cultured on $\mathrm{P}_{\mathrm{DL}} \mathrm{LA}$ (for actual movie, see the main paper Web page).

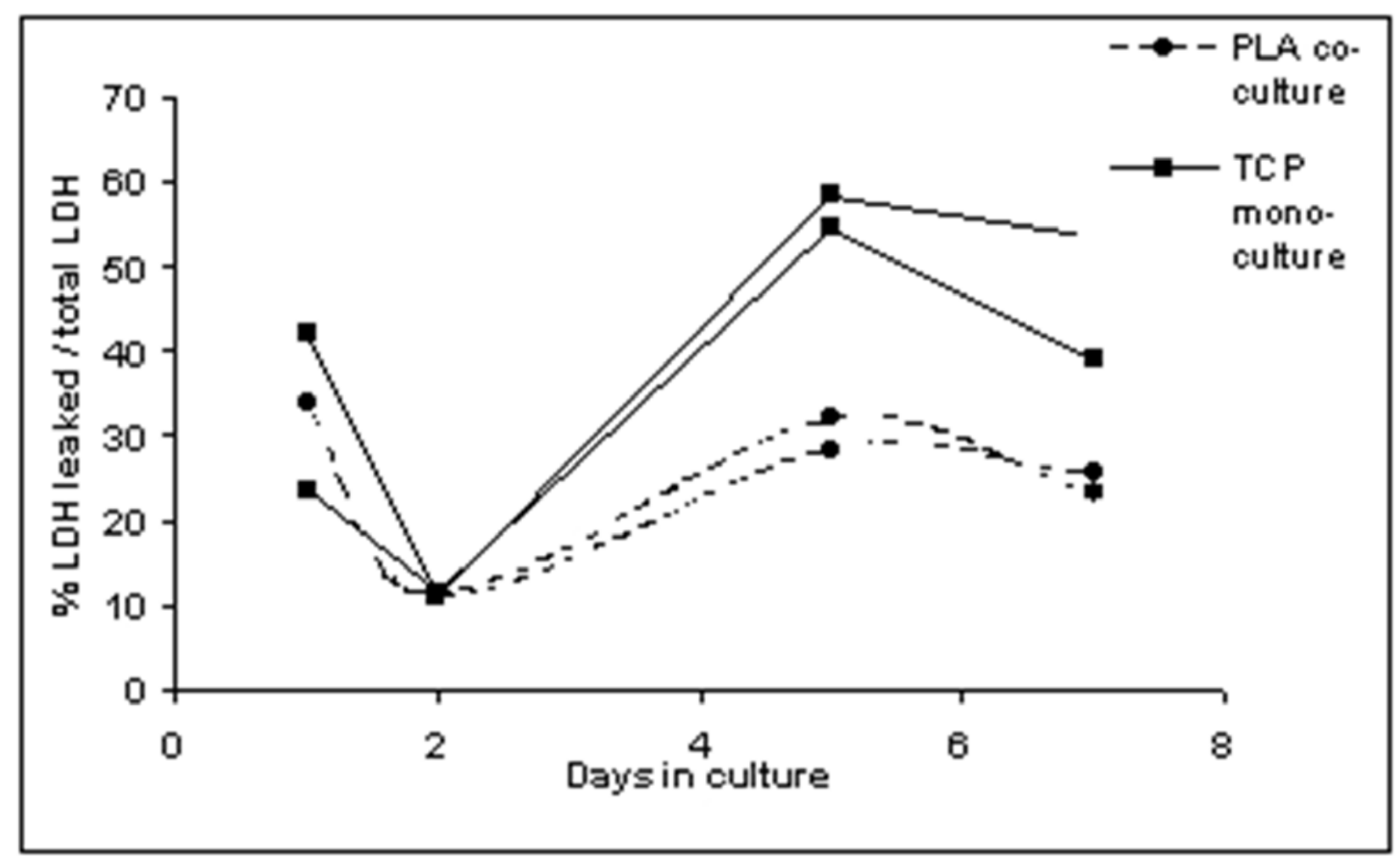

Figure 9: Cell death (assessed as the percentage of LDH leaked as a proportion of the total LDH in the culture well) is similar for both mono-culture and co-culture between 24 and 48 hours at about 10\% (Graph shows two independent experiments). Over the subsequent three days approximately half the cell death occurs in co-culture compared with mono-culture. 

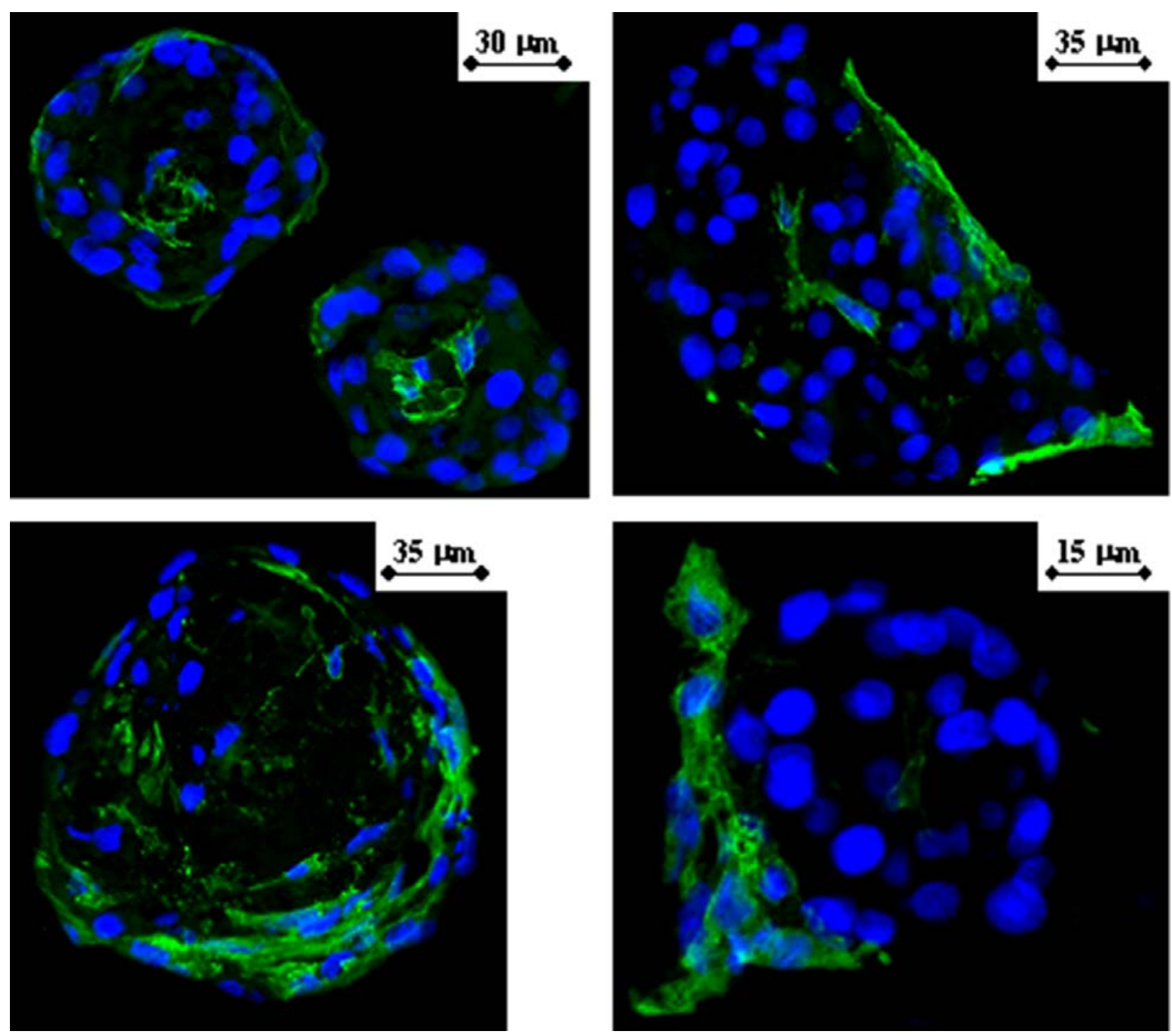

Figure 10: The Stellate cell arrangement around the periphery and in the centre of the co-culture spheroids is shown by FITC immunolocalisation of SMA in spheroid sections prepared after 5 days of co-culture.

sandwich culture system. Toxicol In Vitro 18: 527-532.

Hanada S, Kayano H, Jiang J, Kojima N, Miyajima A, Sakoda A, Sakai Y (2003) Enhanced in vitro maturation of subcultivated fetal human hepatocytes in three dimensional culture using poly-L-lactic acid scaffolds in the presence of oncostatin M. Int J Artif Organs 26: 943951 .

Hasebe Y, Okumura N, Koh T, Kazama H, Watanabe G, Seki T, Ariga T (2005) Formation of rat hepatocyte spheroids on agarose. Hepatol Res 32: 89-95.

Hou DX, Arimura M, Fukuda M, Oka T, Fujii M (2001) Expression of cell adhesion molecule and albumin genes in primary culture of rat hepatocytes. Cell Biol Int 25: 239244.

Koide N, Sakaguchi K, Koide Y, Asano K, Kawaguchi M, Matsushima H, Takenami T, Shinji T, Mori M, Tsuji T (1990) Formation of multicellular spheroids composed of adult rat hepatocytes in dishes with positively charged surfaces and under other non-adherent environments. Exp Cell Res 186: 227-235.

Kudryavtseva EI, Engelhardt NV (2003) Requirement of 3D extracellular network for maintenance of mature hepatocyte morphology and suppression of alphafetoprotein synthesis in vitro. Immunol Lett 15: 25-31.

Powers MJ, Janigian DM, Wack KE, Baker CS, Beer Stolz D, Griffith LG (2002) Functional behavior of primary rat liver cells in a three-dimensional perfused microarray bioreactor. Tissue Eng 8: 499-513.

Riccalton-Banks L, Bhandari R, Fry J, Shakesheff KM (2003) A simple method for the simultaneous isolation of stellate cells and hepatocytes from rat liver tissue. Mol Cell Biochem 248: 97-102.

Sato M, Suzuki S, Senoo H (2003) Hepatic stellate cells: unique characteristics in cell biology and phenotype. Cell Struct Funct 28: 105-112.

Schmeichel KL, Bissell MJ (2003) Modeling tissuespecific signaling and organ function in three dimensions. J Cell Sci 116: 2377-2388.

Seglen PO (1976) Preparation of isolated rat liver cells. Methods Cell Biol 19: 29-83.

Semler EJ, Ranucci CS, Moghe PV (2000) Mechanochemical manipulation of hepatocyte aggregation 
can selectively induce or repress liver-specific function. Biotechnol Bioeng 69: 359-69.

Thomas R, Bhandari R, Barratt D, Bennett A, Fry J, Powe D, Thomson B, Shakesheff KM (2006) The effect of three-dimensional co-culture of hepatocytes and hepatic stellate cells on key hepatocyte functions in vitro. Cells Tissues Organs In press.

Zeilinger K, Holland G, Sauer IM, Efimova E, Kardassis D, Obermayer N, Liu M, Neuhaus P, Gerlach JC (2004) Time course of primary liver cell reorganization in three-dimensional high-density bioreactors for extracorporeal liver support: an immunohistochemical and ultrastructural study. Tissue Eng 10: 1113-1124.

\section{Discussion with reviewers}

Reviewer 1: Measuring viability by the leakage of $\mathrm{LDH}$ activity does not tell us anything about where the cell death has occurred in the spheroid. Do you have any information on this? Is the LDH coming from the stellate cells or the hepatocytes- what kind of cell is dying? Are the cells in the middle of the spheroid dead more often than those on the periphery? Is the spheroid size approaching the limit of oxygen/nutrient permeation?

Is the spheroid size self-limiting, or does it go on increasing if given the opportunity?

Authors: The confocal microscopy and haemotoxylin and eosin stained sections (not shown) were aimed to help identify cell type and position. Both did not show any pattern of cell death and no central necrosis. According to Fukuda et al., 2004, a depth of $80-100 \mu \mathrm{m}$ is the maximum at which hepatocytes can survive in an aggregate structure suggesting our co-culture spheroids are not limited by size.
However, this range will inevitably depend on the density and composition of the aggregate amongst other factors, and we acknowledge that central necrosis is commonly observed if spheroid cultures are maintained beyond a week and this may also occur in this case. Finally, the spheroid size appears to be self limiting. Spheroids formed repeatedly within a similar size range and then remained stable over the culture period.

Reviewer 2: How does the choice of polymer substrate affect the behaviour of the cells in culture? Have the authors compared the results on PDLLA with those on tissue culture plastic?

Authors: The $\mathrm{P}_{\mathrm{DL}}$ LA surface is optimised for dynamic interaction relative to tissue culture plastic. On tissue culture plastic both cell types attach to the surface to form a mono-layer. Retraction of stellate cell processes is not seen and cell contact is limited to the positions in which the cells initially attach.

Reviewer 3: The authors may want to relate the arrangement of the cells inside the spheroids and the size of the spheroids to the in vivo situation.

Whilst 3D aggregates are formed, the cell culture surface is still 2D. Could the authors comment on whether they expect differences between the 2D culture on PLA surfaces and 3D culture on polymer scaffolds.

Authors: The spheroids are not in vivo like other than the $3 \mathrm{D}$ contacts they allow between the stellate cells and the hepatocytes. We cannot speculate on the effect of a 3D scaffold. However, such a system may allow the spatial aspects of the interaction to be controlled and further investigated. 